\title{
New Technology Foresight Method: Extenics, Extension Engineering method and Technology ----Second Creation
}

\author{
Zhao LIU ${ }^{1, a}$, Mei ZHANG ${ }^{2, b}$, Xia XU $U^{3, c}$ \\ ${ }^{1}$ Department of Computer Science, Guangdong University of Technology, \\ Guangzhou, 510006, China \\ ${ }^{2}$ Department of Computer Science, Guangdong University of Technology, \\ Guangzhou, 510006, China \\ ${ }^{3}$ Zhongkai University of Agriculture and Engineering, \\ Guangzhou, 510006, China \\ aemail: 190381200@qq.com, bemail: 646054552@qq.com, cemail: Iz8797483@163.com
}

Keywords: Technology Foresight; Extension Theory; Artificial Intelligence

\begin{abstract}
With the speed of the development of science and technology speeds up unceasingly, the traditional technology forecasting often limited the price of large, long cycle. This paper proposes a new subject to carry out technology foresight and industrial path planning research---extenics. Extension through the study on the law of the development of things development, established to deal with the contradictions in the development of the method system of and of technology foresight subject in the problem of intelligent processing, the system must also extent make up the including the Delphi method, the traditional forecasting methods lack of trapped.
\end{abstract}

\section{Introduction}

The development of science and technology has broken through the limitation of the traditional understanding of human being, and has led to a new scientific and technological revolution, and the trend of the integration of science and technology is becoming more and more obvious. Between subjects, science and technology, natural science and Humanities and Social Sciences cross penetration, leading to the birth of a new field of interdisciplinary, showing the trend of community breakthrough. In today's world and between countries and within countries, each level of the field of technology foresight activities appeared a lot of new content, are increasingly using many new methods, from its mission and role to the system and the method have undergone many changes. In particular, the emergence of new methods, new models greatly improve the ability and level of technology foresight activities. But the traditional methods are often limited by economic and time periods. Extension objects of study are contradictory problems, the basic theory is the extension theory, method system is can extension method (because these methods are particularly suitable for innovation, so also known as extension method innovation), the logical basis is extension logic. In the field with the cross is formed by the fusion of extension engineering. Extenics specific strategies are dynamically updated and due to its formal and qualitative and quantitative combination, avoid the traditional technology and forecast the transformation characteristics of mathematical modeling methods often the actual content of the rounding the disadvantages, and the contradictions and problems of the shortcoming of the traditional technological prediction were to a certain extent make up. [1-5].

\section{Form of Technology Foresight Limitations of the method}

Because of the different emphasis and research direction, the domestic and foreign have different definitions and explanation of the "technology foresight". But according to the official national technology foresight projects and developed countries of professional papers, by the University of 
Sussex SPRU (Science Policy Research Unit) Policy Research Institute of Ben R. Martin Professor definition of technology foresight is being widely accepted by all countries in the, he in the middle of the 1990s put forward the concept: "the belief that technology foresight is the future for a long period of scientific, technical, economic and social development of the contribution of the general technology [4]".

Although the technology foresight in this research project, because of its special development constraints which began in developed countries since the middle of last century, the United States, but in fact the earliest carry out with the scale and continued to carry out technology foresight project carried out by the country is Japan. Japan since 1970s, the organization of a large-scale multi subject technology foresight survey every five years. And Europe in the early ninety's after the turmoil followed by Japan to carry out technical foresight project to carry out [5]. So we can attribute the technology foresight experience from the United States to Japan to promote the development of Europe and the United States to join the historical process and mode. And to the understanding in the nineties of the twentieth century, because of the Asia Pacific Economic Cooperation (APEC), involved in the organization of international economic cooperation and Development Organization for economic co operation and development (OECD) and the United Nations Industrial Development Organization (UNIDO), technology foresight is promoted to a new stage. Since then, technology foresight has become a major feature and trend of the development of the world's science and technology in twenty-first Century. China complies with international technology development trend, at the beginning of the 20th century to carry out the system of technology foresight activities: the Chinese Academy of Sciences in August 2003 started "in China over the next 20 years of Technology Foresight Research", anticipate research methods, technical requirements, study, Delphi survey, policy from the technical analysis, technology development trend tracking and monitoring of five direction began to study [6-7].

Delphy method is the most commonly used and effective method in the research of technology foresight. Delphy method is a "non - meet - type" expert collection methods and "a highly efficient, through group communication and communication to solve the complex problem of the method" [4]. Delphy method essence is a kind of subjective forecasting method based on the technology. Each of the steps are required to follow the scientific method, especially in dealing with a large number of questionnaire, itself is using the professional statistics and economics knowledge, which makes the statistics of the with the objective factors, quantitative description of qualitative investigation of the topic of the research, the conclusions more scientific. But due to the following reasons, making the Delphy method in the second industry to develop the limitations of industrial planning route very prominent

(1), it need a lot of resources be devoted to (investigation), (organized a team of experts) manpower and material resources (workload), making a number of small and medium-sized enterprises can not afford the cost of;

(2). Instant technology can be realized, because of social development and resistance factors and the technology foresight results in the actual utilization ratio is very low. Figure 1 shows that the eighth technology foresight project is expected to achieve the time distribution, we can see from the chart that Japan is now the technology foresight project results in the actual use of the effect is not obvious

.(3). Most of the traditional technology forecasting methods are static, one-time prediction, this model can not adapt to the current situation of the development of social science and technology is changing rapidly. 
Figure 1: Technology forecasting stability of each cycle

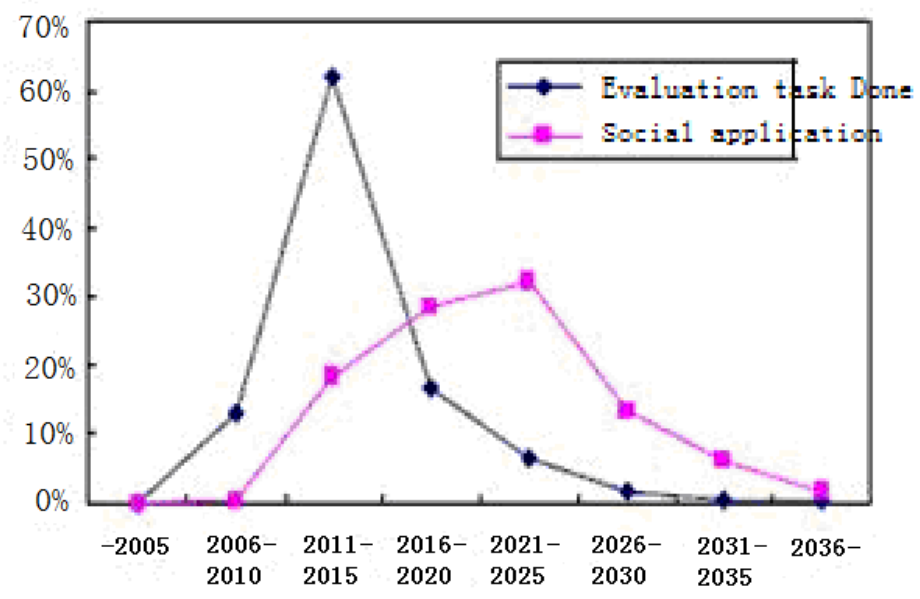

\section{Extension engineering method and extension design method second creation method}

Extension design method in the second creation method, its core thinking for prediction technology subject which, we can be described in this way: from a or a plurality of mature products or technology of the transform it (them) parts of the physical elements, and construct a new technology or new technology development direction. The creation method is in line with the technology forecast method.[6-7]

Second creation method is following this law:

(1) The basic elements of the decomposition product technology

Decomposition of the original product $O$, list the actual and actual parts, hard and soft parts, the latent and negative positive. In some electronic products or mechanical products that require bearing assembly, we also need to list their intermediary department.

(2) List the main features of the original product $O$ and its conjugate

List the main features of the $O$ conjugate $\left\{t_{f}\right\}$, the performance characteristics of $\left\{t_{g}\right\}$ and the real meaning of $\left\{t_{r}\right\}$.

(3) Intelligent calculation of its corresponding value.

(4)Description of the origin of $\mathrm{O}$ different levels of matter element $M, M_{i}, M_{i j} \ldots$ ( $\mathrm{i}=1,2, \ldots \mathrm{n} ; \mathrm{j}$ $=1,2, \ldots, \mathrm{m})$

Obviously

$$
\begin{aligned}
& M=\left[\begin{array}{ccc}
O, & c_{1}, & V_{1} \\
& c_{2}, & V_{2} \\
\square & \square \\
& c_{n} & V_{n}
\end{array}\right], M_{i}=\left[\begin{array}{ccc}
O_{i}, & c_{1}, & V_{i 1} \\
& c_{2}, & V_{i 2} \\
& \square & \square \\
& c_{n} & V_{i n}
\end{array}\right], \\
& M_{i j}=\left[\begin{array}{ccc}
O, & C_{1}, & V_{i j 1} \\
& C_{2}, & V_{i j 2} \\
& \square & \square \\
C_{n} & V_{i j n}
\end{array}\right], \ldots
\end{aligned}
$$

$M=\prod_{i=1}^{n} M_{i}=\prod_{i=1}^{n} \prod_{j=1}^{m} M_{i j}=\ldots$

(5) The matter element transformation

The transformation and combination of the various elements obtained from the step (4) to generate a variety of new functions and functional values, in order to conceive a new product (or product technology concept). The basic form of transformation is the replacement, decomposition, additions and deletions and expanding and shrinking by of with information elements according to 
the basic transform, transform operation by intelligent machine learning can obtain new products (or technical) a variety of programs:

$T M=\prod_{i=1}^{n} T_{i} M_{i}=\prod_{i=1}^{n} \prod_{j=1}^{m} T_{i j} M_{i j}=\ldots$

(6) Preliminary evaluation

(7) The idea of a new product (or technology of the conjugate part)

(8) To re select the appropriate basis elements as parts

(9) Repeat the above steps, evaluation and revision, and finally come to the technical prediction

\section{Conclusion}

In this paper, the shortcomings of the traditional technology forecasting methods --- "cost big and long cycle" as the cut in, put forward the use of new disciplines, "extension" for the technical prediction of the research and development of the subject. The "second creation method" of the extension design method is used to supplement and perfect the concept and method of modern prediction. New possibilities for technology forecasting and industrial planning of small and medium sized enterprises are presented.

\section{Acknowledgement}

In this paper, the research was sponsored by the Natural Science Foundation of Guangdong Province (Project No. S2011040004281, S2013010014457).

\section{References}

[1] WANG Jin-bo. Technology Foresight: science and technology strategic planning and science and technology policy development [J]. China Soft Science , 2004, fifth (5): 62-66.

[2] PU Gen-xiang et al. On the basic assumption of the theory of Technology Foresight: the research of [J]. natural dialectics , 2006, 18 (7): 40-43.

[3] LI Guo-qiu,LONG Yi. Nearly a decade (2004 to 2013) International Technology Foresight research focus and Trend Analysis [J]. Document, Information \& Knowledge, 2014, 3 (3): 104-116.

[4] LI Guo-qiu,WANG Xiao-yun. Technology Foresight applied to predict the market feasibility analysis and implementation process [J]. Library Journal, 2014, 1 (1): 8-19.

[5] Cai Wen, Yang Chunyan, \& He Bin. (2003) new development of basic theory of extension science. Chinese Engineering Science, 5 (2), 80-87.

[6] Yang Chunyan, Wang Guanghua, Cai Wen. A new cross subject -- extension [J]. China Science Foundation, 2004, 18 (05): 268-272.

[7] Chen Shouyu. Variable fuzzy set theory -- Also on the mathematics and logic error of extension theory [J]. Journal of Dalian University of Technology, 2007, 47 (4): 618-624. 